\title{
Interface Engineering in Heteroepitaxy
}

\author{
S.K. Hong, Y. Chen, H.J. Ko and T. Yao \\ Institute for Materials Research, Tohoku University, Sendai 980-8577, Japan
}

\begin{abstract}
We report the importance of interface engineering in heteroepitaxy with examples of plasma-assisted molecular beam epitaxial $\mathrm{ZnO}$ growths on (0001) sapphire substrates and on (0001) GaN/sapphire templates, whose interfaces are engineered to improve and to control properties of $\mathrm{ZnO}$ films. The growth of rocksalt structure $\mathrm{MgO}$ buffer on $\mathrm{Al}_{2} \mathrm{O}_{3}$ (0001) is developed for $\mathrm{ZnO}$ epitaxy. By employing the $\mathrm{MgO}$ buffer layer, the formation of $30^{\circ}$ rotated mixed domains is prohibited and two-dimensional layer-by-layer growth of $\mathrm{ZnO}$ on sapphire substrate is achieved. High-resolution X-ray diffraction reveals the superior improvement in a crystal quality of $\mathrm{ZnO}$ films with an $\mathrm{MgO}$ buffer. Polarity of wurtzite structure $\mathrm{ZnO}$ films on Ga-polar GaN/sapphire templates is controlled by changing interface structures. By forming a single crystalline, monoclinic $\mathrm{Ga}_{2} \mathrm{O}_{3}$ interfacial layer between $\mathrm{GaN}$ and $\mathrm{ZnO}$ through O-plasma pre-exposure on the Ga-polar GaN surface, O-polar ZnO films are grown. By forming the $\mathrm{ZnO} / \mathrm{GaN}$ heterointerface without an interfacial layer through the $\mathrm{Zn}$ pre-exposure on the Ga-polar GaN surface, Zn-polar ZnO films are grown.
\end{abstract}

PACS numbers: 68.35.-p, 68.35.Dv, 81.15.-z, 81.15.Hi, 81.10.Aj

\section{Introduction}

$\mathrm{ZnO}$, one of wide band gap semiconductors, has been revealed that this material is promising for exciton-based photonic devices in the ultra-violet region [1-3]. The absence of a suitable substrate for $\mathrm{ZnO}$ epitaxy is an emerging issue in $\mathrm{ZnO}$ epitaxy. Homoepitaxy may provide a promising solution for this issue and commercial $\mathrm{ZnO}$ substrates are being supplied. Several benefits of homoepitaxy, such as (1) perfect lattice matching, (2) no thermal mismatch, (3) no crystallographic mismatch, and (4) simple control of lattice polarity by using different-polar substrates, are expected. However, crystal qualities of the $\mathrm{ZnO}$ substrate are far from those applicable to $\mathrm{ZnO}$ epitaxy for devices [4].

$\mathrm{Al}_{2} \mathrm{O}_{3}$ (0001), one of the most popular oxide substrates, has widely been used for the substrate for $\mathrm{ZnO}$ epitaxy. However, there are considerable mismatches in this heteroepitaxy system including large lattice misfit of $18.6 \%$, crystallographic 
mismatch, and thermal mismatch. Those mismatches have caused various problems including a highly disordered heterointerface, a formation of $30^{\circ}$-rotated domains, and degraded crystallinity of $\mathrm{ZnO}$ layers [5]. Recent achievements in heteroepitaxy of wide band gap semiconductors on highly mismatched substrates largely rely on how well a template is created in between the epilayer and the substrate [6]. Therefore, the exploring and development of epitaxy technique, which can provide a suitable template for $\mathrm{ZnO}$ epitaxy, are highly needed for $\mathrm{ZnO}$ epitaxy on $c$-plane sapphire considering the mismatches in the system.

As a non-oxide substrate, $\mathrm{GaN}$ is an attractive material applicable as a substrate for $\mathrm{ZnO}$ epitaxy. In the case of a $\mathrm{ZnO} / \mathrm{GaN}$ system, there are several merits compared with the $\mathrm{ZnO}$ /sapphire: (1) smaller lattice misfit (1.9\%), (2) no crystallographic mismatch, and (3) smaller thermal mismatch. Although there are such merits in using $\mathrm{GaN}$ as a substrate for the $\mathrm{ZnO}$ epitaxy, the getting of the high quality GaN substrates is as difficult as the case for $\mathrm{ZnO}$. Fortunately, the recent progress in metal organic chemical vapor deposition (MOCVD) of GaN on c-plane sapphire can provide a relatively high quality of GaN template substrate on sapphire, which can be used as a substrate for $\mathrm{ZnO}$ epitaxy. Because the polarity of such high-quality MOCVD GaN is typical of Ga-polar and both $\mathrm{ZnO}$ and $\mathrm{GaN}$ are of wurtzite structure, the polarity of $\mathrm{ZnO}$ films on GaN may reflect the polarity of underlying GaN. Therefore, a selective growth of $\mathrm{Zn}$ - and O-polar $\mathrm{ZnO}$ films on Ga-polar GaN is an attractive issue for the progress of heteroepitaxy in material systems of wurtzite structure.

In this article, the importance of interface engineering in heteroepitaxy is demonstrated, the concept of which can be applied to other material systems, with our recent results of (1) drastic improvement in crystal qualities of $\mathrm{ZnO}$ films on $c$-plane sapphire by using a novel $\mathrm{MgO}$ buffer layer $[7,8]$ and (2) control of $\mathrm{ZnO}$ film polarities on Ga-polar GaN templates by controlling interface formations [9, 10].

\section{Experimental}

\section{1. $\mathrm{ZnO}$ on $\mathrm{Al}_{2} \mathrm{O}_{3}(0001)$}

Commercial $\mathrm{Al}_{2} \mathrm{O}_{3}$ (0001) substrates were used. Reflection high-energy electron diffraction (RHEED) was used to monitor the growth procedures. The deposition of $\mathrm{MgO}$ buffer layer is carried out at $480^{\circ} \mathrm{C}$ with a slow deposition rate of about $0.01 \AA / \mathrm{s}$ under oxygen rich conditions. We found that a low growth rate is critical to the formation of an $\mathrm{MgO}$ wetting layer on the $\mathrm{Al}_{2} \mathrm{O}_{3}$ (0001) surface. After the deposition of a nominal $2 \mathrm{~nm}$ thick $\mathrm{MgO}$ buffer, $\mathrm{ZnO}$ growth is carried out at $480^{\circ} \mathrm{C}$ for a few minutes and an annealing at $750^{\circ} \mathrm{C}$ under the exposure of oxygen plasma for 5 minutes is performed. The subsequent growth of the $\mathrm{ZnO}$ is then carried out at a temperature of $650^{\circ} \mathrm{C}$, with a $\mathrm{Zn}$ beam flux around $0.22 \mathrm{~nm} / \mathrm{s}$ and oxygen flow rate of $1.5 \mathrm{sccm}$ under the plasma power of $300 \mathrm{~W}$. RHEED inten- 
sity oscillations using the specular spot can be recorded during this growth stage. $\mathrm{X}$-ray diffraction (XRD) was used to investigate the structural properties of the $\mathrm{ZnO}$ epilayers. To directly measure the buffer, thin $\mathrm{ZnO}$ layers were grown and grazing incident XRD was used for characterization.

\subsection{ZnO on GaN template}

$4 \mu \mathrm{m}$-thick Ga-polar GaN epilayers grown by MOCVD on $\mathrm{Al}_{2} \mathrm{O}_{3}(0001)$ were used as templates for $\mathrm{ZnO}$ epitaxy. The $\mathrm{GaN}$ template was thermally cleaned at $700-750^{\circ} \mathrm{C}$ in ultra-high vacuum for about $1 \mathrm{~h}$ after being degreased in acetone and methanol. The Zn flux was set to about $0.15-0.20 \mathrm{~nm} / \mathrm{s}$ and O-plasma conditions were set to $300-400 \mathrm{~W}$ of power with an oxygen gas flow rate of $3.5 \mathrm{sccm}$. The growth under these conditions gave a flux ratio that was oxygen rich. Two types of pre-growth treatments of the GaN surface were examined: (1) Zn pre-exposure for 2-3 min, and (2) O-plasma pre-exposure for $3-5 \mathrm{~min}$. The interface structure was observed by high-resolution transmission electron microscopy (HRTEM) with

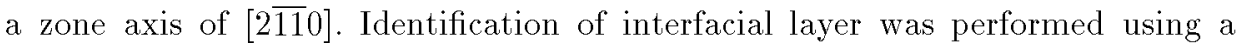
digital diffraction pattern (DDP) obtained by fast Fourier transform (FFT) of the HRTEM image. The polarity of the $\mathrm{ZnO}$ films was determined by convergent beam electron diffraction (CBED). The CBED patterns were obtained at $100 \mathrm{keV}$

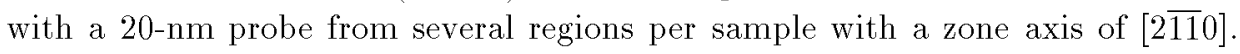
Experimental CBED patterns from the $\mathrm{ZnO}$ and GaN layers were compared with simulated patterns for TEM specimen thicknesses of 10-80 nm to determine the polarity.

\section{Results and discussion}

\subsection{ZnO films with $\mathrm{MgO}$ buffer on c-plane sapphire}

Figure 1 shows the RHEED patterns recorded during the initial growth stages. At the very beginning of $\mathrm{MgO}$ deposition, a diffuse streaky pattern (Fig. 1a) evolves indicating the formation of an $\mathrm{MgO}$ wetting layer on the $\mathrm{Al}_{2} \mathrm{O}_{3}(0001)$ surface. According to the growth rate the evaluated thickness is between one and two monolayers. The continuing growth of MgO leads to diffused spotty patterns indicative of a transition to the island growth mode (Fig. 1b), which is caused by the near 9\% in-plane lattice mismatch if $\mathrm{MgO}$ grows with its [111] axis normal to the $\mathrm{Al}_{2} \mathrm{O}_{3}$ (0001) plane. As the $\mathrm{ZnO}$ deposition starts, spotty patterns (Fig. 1c) with a rod spacing of about $9 \%$ smaller emerge superimposing on the $\mathrm{MgO}$ patterns. As the growth is continuing, $\mathrm{ZnO}$ patterns gradually take the places of the $\mathrm{MgO}$ pattern, and of the same time, the RHEED spots elongate and connect with each other. Finally, streaky RHEED patterns evolve. Such a procedure takes about one hour, corresponding to the deposition of a 200-300 nm thick film. In another approach, annealing at $700^{\circ} \mathrm{C}$ for about $5 \mathrm{~min}$ is carried out on the low tem- 
perature grown $\mathrm{ZnO}$ layer with a thickness of around several nanometers. Sharp streaky RHEED patterns (Fig. 1d) were present after annealing. Comparing with the growth of $\mathrm{ZnO}$ on $\mathrm{Al}_{2} \mathrm{O}_{3}$ (0001) without the use of an $\mathrm{MgO}$ buffer, where a columnar growth usually takes place, the use of a $\mathrm{MgO}$ buffer successfully promotes the lateral growth of $\mathrm{ZnO}$ at an initial stage and a flat $\mathrm{ZnO}$ template is created for subsequent two-dimensional (2D) $\mathrm{ZnO}$ growth.
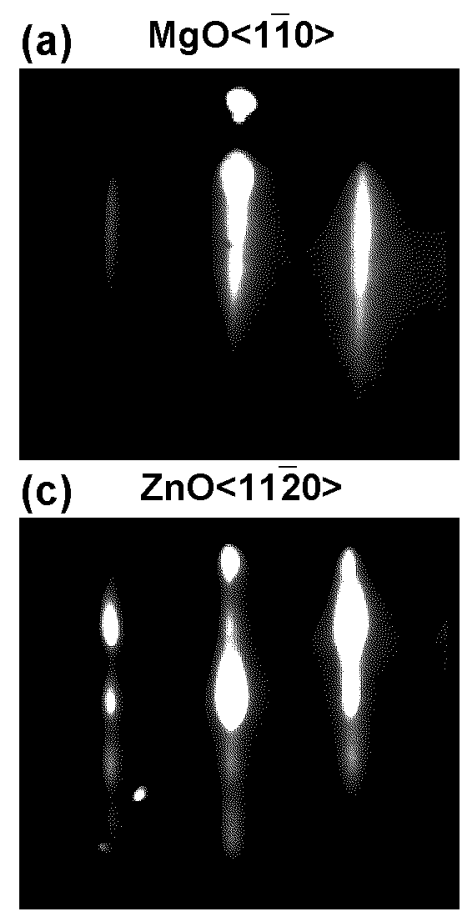

(b) $\mathrm{MgO}<1 \overline{10}>$

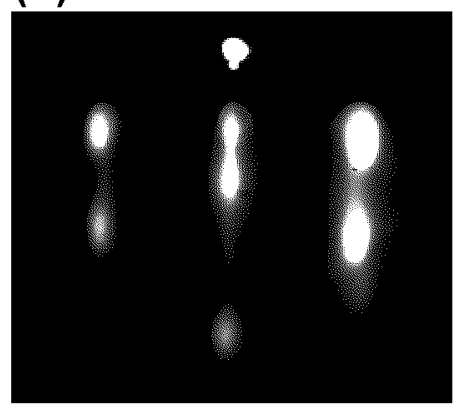

(d) $\mathrm{ZnO}<11 \overline{20}>$

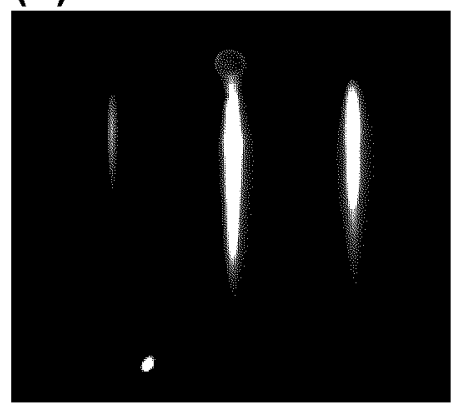

Fig. 1. RHEED patterns at different growth stages: (a) the $\mathrm{MgO}$ wetting layer on $\mathrm{Al}_{2} \mathrm{O}_{3}$ (0001) substrate, (b) the $\mathrm{MgO}$ buffer layer after a 2D-3D transition, (c) the $\mathrm{ZnO}$ layer grown at $480^{\circ} \mathrm{C}$ on the $\mathrm{MgO}$ buffer, (d) the $\mathrm{ZnO}$ layer after annealing at $750^{\circ} \mathrm{C}$ for $5 \mathrm{~min}$.

As an evidence of 2D layer-by-layer growth, RHEED intensity oscillations are recorded during the consequent $\mathrm{ZnO}$ growth. Figure 2 shows the RHEED specular beam intensity oscillations with the incident electron beam along the $\mathrm{ZnO}$ $\langle 11 \overline{2} 0\rangle$ azimuth at various growth temperatures. More than fifty oscillations are recorded at the growth temperature of $400^{\circ} \mathrm{C}$, while only a few slight oscillations can be observed at $720^{\circ} \mathrm{C}$. The fast damp of the RHEED oscillations at a higher growth temperature is due to the growth mode transition from $2 \mathrm{D}$ nucleation to step-flow mode but not to the surface morphology degrading, since the sharp streaky RHEED pattern with the intense specular spot becomes even clearer at higher temperatures. 


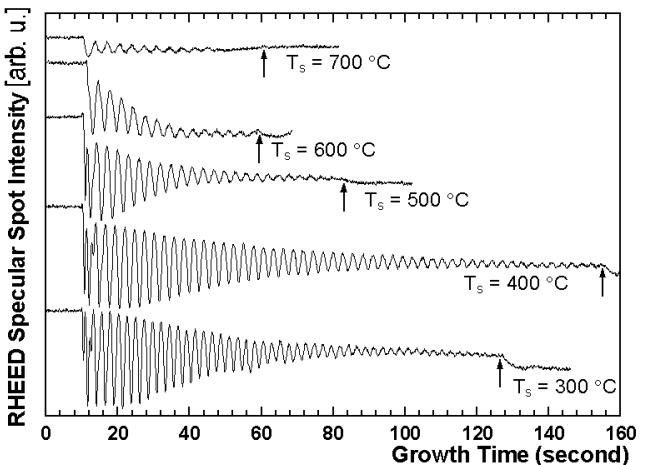

Fig. 2. RHEED specular spot intensity oscillations on the $00 \operatorname{rod}$ in $\langle 11 \overline{2} 0\rangle$ azimuth at various growth temperatures. Arrows indicate where the growth is interrupted.
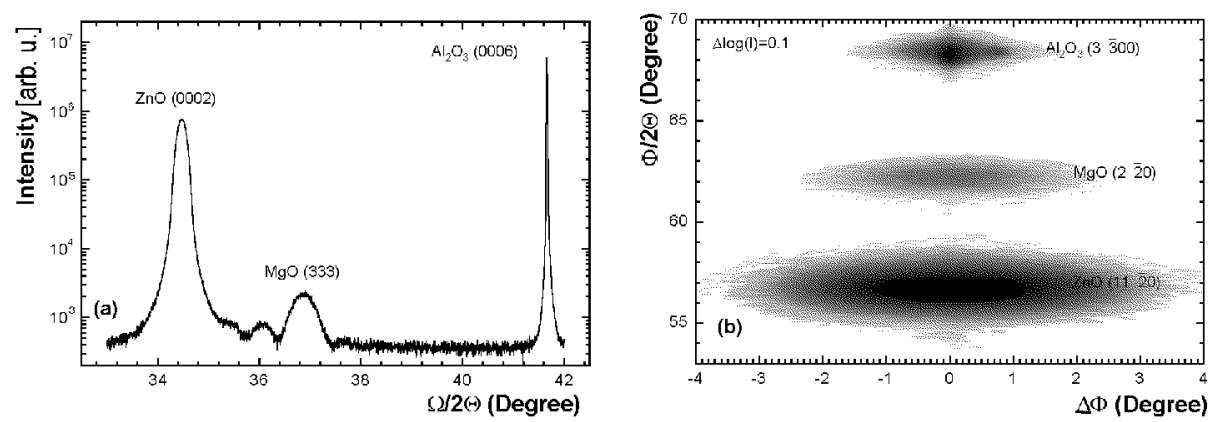

Fig. 3. (a) $\Omega-2 \Theta$ scan along the surface normal of a thin $\mathrm{ZnO}$ epilayer grown on $\mathrm{Al}_{2} \mathrm{O}_{3}$ (0001) with an MgO buffer; (b) Grazing-indent XRD mapping of the in-plane diffraction peaks.

In order to identify the crystal structure of the buffer, an $\mathrm{MgO}$ layer about $7 \mathrm{~nm}$, thicker than a usually used $2-3 \mathrm{~nm}$ buffer, was grown and covered by a $15 \mathrm{~nm}$ thick $\mathrm{ZnO}$ layer. The growth processes monitored by RHEED did not show any difference from a typical buffer, which allows us to use this sample for investigating the crystal structure of the $\mathrm{MgO}$ buffer. Figure 3 a shows the XRD $\Omega-2 \Theta$ scan along the surface normal of the sample. Besides the $\mathrm{ZnO}(0002)$ and the $\mathrm{Al}_{2} \mathrm{O}_{3}(0006)$ peaks, a diffraction peak at $2 \Theta=36.910^{\circ}$ is observed. After correcting the zero-error of the XRD diffractometer, an inter-plane distance of $0.2434 \mathrm{~nm}$ is obtained. This value corresponds to the distance between (333) planes in bulk $\mathrm{MgO}$ with a strain less than $0.07 \%$ indicating the fully relaxed $\mathrm{MgO}$ growth along [111] direction on $\mathrm{Al}_{2} \mathrm{O}_{3}$ (0001) substrate. The fringes confirm the thickness of the $\mathrm{MgO}$ layer of about $7 \mathrm{~nm}$. The presence of interference fringes also indicates a sharp interface with little inter-diffusion. To get further information of the structural properties, grazing-incident XRD measurements were carried out. Figure 3b 
shows the in-plane reciprocal space mapping. The rocksalt $\mathrm{MgO}(2 \overline{2} 0)$ peak appears in alignment with $\mathrm{ZnO}(11 \overline{2} 0)$ and $\mathrm{Al}_{2} \mathrm{O}_{3}(3 \overline{3} 00)$. Thus the in-plane epitaxial relationship is determined as $\mathrm{ZnO}[1 \overline{1} 00] / \mathrm{MgO}[1 \overline{1} 0] / \mathrm{Al}_{2} \mathrm{O}_{3}[11 \overline{2} 0]$, consistent with the RHEED observation [7]. No other structural phases were observed by grazing-incident XRD measurements. Therefore, we can exclude the alloy formation from $\mathrm{Al}_{2} \mathrm{O}_{3} / \mathrm{MgO} / \mathrm{ZnO}$ interfaces. We know that the oxygen sub-lattice with hexagonal $\left(\mathrm{ZnO}\right.$ and $\left.\mathrm{Al}_{2} \mathrm{O}_{3}\right)$ or cubic $(\mathrm{MgO})$ close-packed structure determines the lattice matching of two oxides. Therefore, the lattice mismatch is $18.6 \%$ and $8.3 \%$ for $\mathrm{ZnO}(0001) / \mathrm{Al}_{2} \mathrm{O}_{3}(0001)$ and $\mathrm{MgO}(111) / \mathrm{Al}_{2} \mathrm{O}_{3}(0001)$, respectively. The metal cations occupy either the tetragonal ( $\mathrm{Zn}$ in $\mathrm{ZnO}$ ) or the orthogonal ( $\mathrm{Al}$ in $\mathrm{Al}_{2} \mathrm{O}_{3}$, and $\mathrm{Mg}$ in $\mathrm{MgO}$ ) vacancy formed by oxygen anions, which leads to a chemical mismatch across the hetero-interface. The rocksalt MgO finds its in-plane lattice constant just at the intermediate position between that of $\mathrm{ZnO}$ and $\mathrm{Al}_{2} \mathrm{O}_{3}$, as well as its poly-covalent ionic bonding situation. This may explain why $\mathrm{MgO}$ wets $\mathrm{Al}_{2} \mathrm{O}_{3}$ and $\mathrm{ZnO}$ adhere to $\mathrm{MgO}$. We should clarify that no improvement in morphology and crystal quality can be obtained if $\mathrm{ZnO}$ is grown directly on an $\mathrm{MgO}$ wetting layer. This is reasonable because the release of strain greatly contributes to lowering the surface energy and reducing the mismatch. Furthermore, the 2D-3D transition resulted islands provide nucleation cores for the following $\mathrm{ZnO}$ growth.

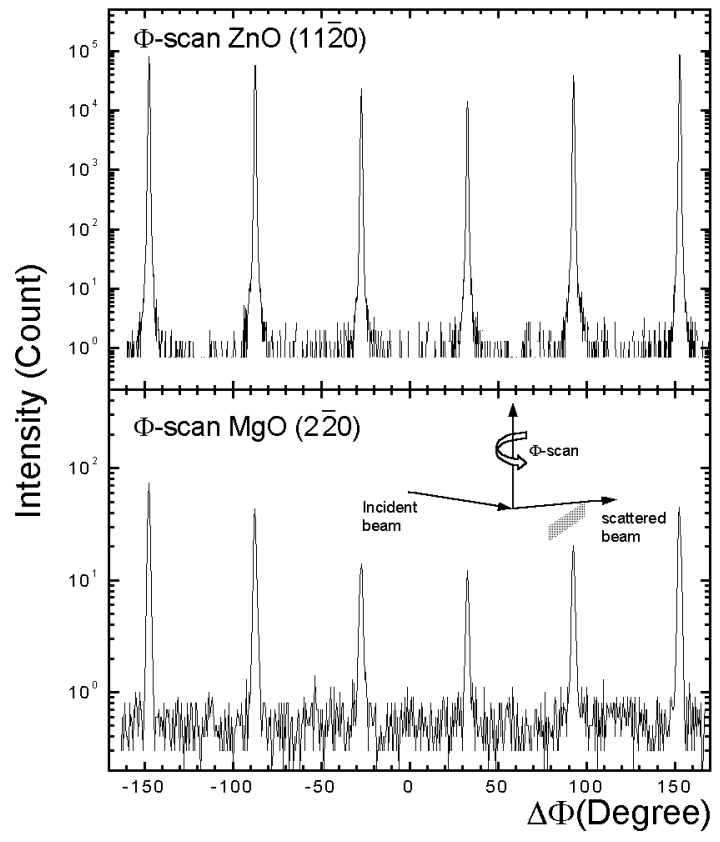

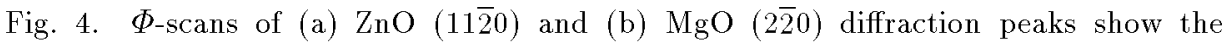
in-plane epitaxial relationship. The insertion in (b) indicates the geometry of scan. 
Figure 4 shows $\Phi$-scan with an incident angle of $0.35^{\circ}$. MgO $(2 \overline{2} 0)$ peaks are found coincident with $\mathrm{ZnO}(11 \overline{2} 0)$, which confirms again that $\mathrm{MgO}$ has a rocksalt structure and has the in-plane epitaxial relationship as $\mathrm{ZnO}[1 \overline{1} 00] / \mathrm{MgO}[1 \overline{1} 0] /$ $\mathrm{Al}_{2} \mathrm{O}_{3}[11 \overline{2} 0]$. From these results, we speculate that the effect of $\mathrm{MgO}$ buffer is mainly in lowering the substrate surface energy, which is favorable to the lateral growth of $\mathrm{ZnO}$. It should be strongly noted that Fig. 4 indicates six-fold rotational symmetry along the $\langle 0001\rangle$ direction, which means there are no formation of $30^{\circ}$ rotated mixed domains.

In Fig. 5 we compare the high-resolution XRD results taken from the $\mathrm{ZnO}$ epilayers grown on $\mathrm{Al}_{2} \mathrm{O}_{3}$ with and without an $\mathrm{MgO}$ buffer. Figure 5 a shows the $\Omega-2 \Theta$ scans of (0002) peak. The presence of fringes in the (0002) diffraction peak of the $\mathrm{ZnO}$ epilayer with a buffer indicates a flat surface and interface. This is also confirmed by AFM measurement. For this sample, the root mean square (rms) value of the surface roughness is less than $8 \AA$ over a $10 \mu \mathrm{m} \times 10 \mu \mathrm{m}$ area. The FWHM of the (0002) $\Omega$-rocking-curve (Fig. 5b) of the $\mathrm{ZnO}$ layer with an $\mathrm{MgO}$ buffer gives an extremely small value of 13 arcsecond when comparing with the 774 arcsecond of the layer without $\mathrm{MgO}$ buffer, which indicates finely ordered $c$-planes as a consequence of a well-controlled layer-by-layer growth, and also implies a very low screw dislocation density. For wurtzite material grown along the $c$-axis,
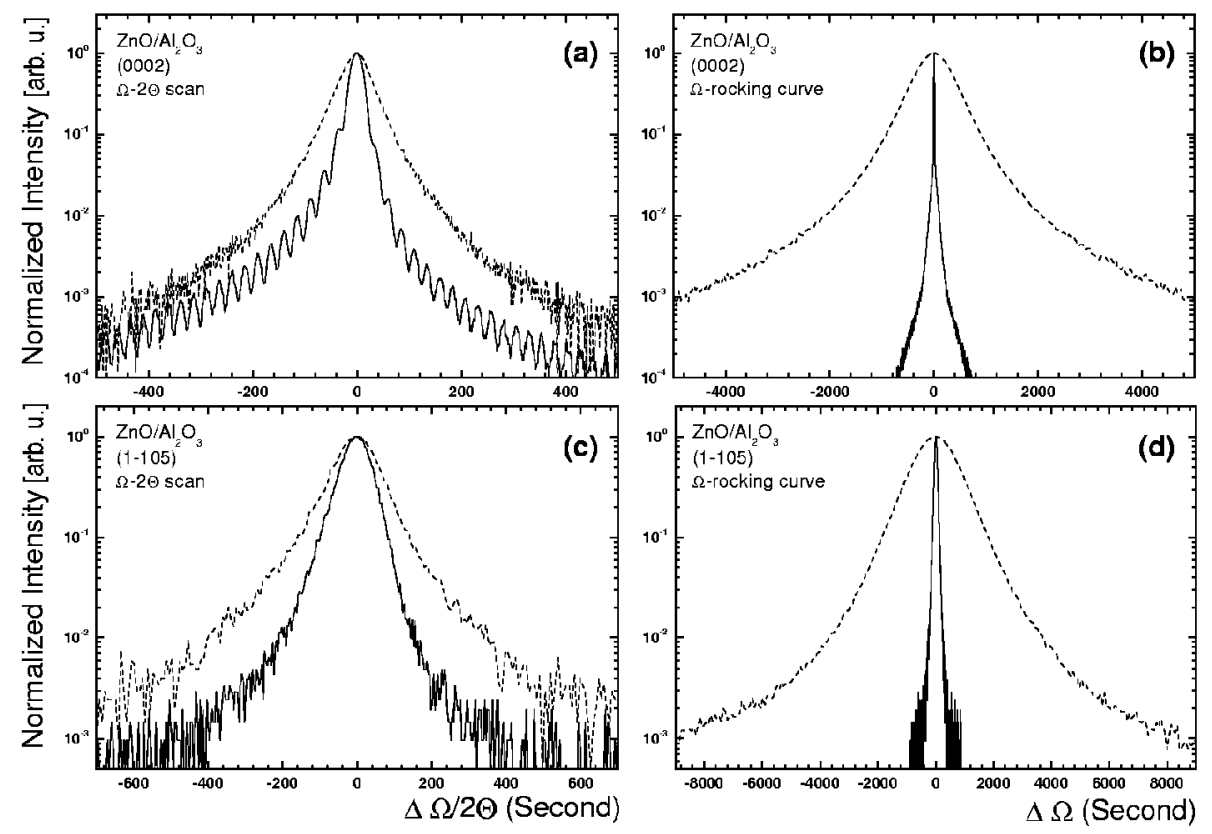

Fig. 5. High-resolution XRD $\Omega$-rocking curves and $\Omega-2 \Theta$ scans of the $\mathrm{ZnO}$ epilayers grown on $\mathrm{Al}_{2} \mathrm{O}_{3}$ with (solid line) and without (dashed line) an $\mathrm{MgO}$ buffer. 
the most favorable dislocation is the pure edge dislocation, which causes little crystallographic tilt in the (0001) planes. In order to obtain the information of total dislocation density we examined the (10 $\overline{1} 5)$ asymmetric diffraction peak of $\mathrm{ZnO}$. A $\Omega$-rocking curve is shown in Fig. 5d. The FWHM is 108 arcsecond for the $\mathrm{ZnO}$ layer with buffer and 1640 arcsecond for the layer without. In the case of $\Omega-2 \Theta$, the differences in peak widths are not as large as the rocking-curves. This is because the dislocations have less effect on the inhomogeneous strain than on the mosaicity. All the XRD values shown here are as good as that of GaN grown by MOCVD on $\mathrm{Al}_{2} \mathrm{O}_{3}$ with a low temperature buffer.

\subsection{Polarity controlled ZnO films on Ga-polar GaN templates}

Since the polarity of the used GaN templates is Ga-polar (cation-polar), the growth of $\mathrm{Zn}$-polar $\mathrm{ZnO}$ films would be possible if we could form "N-Ga-O-Zn" bonding at the interface. However, the formation of such an interface would not be so easy because of reactivity of the GaN surface with oxygen, which leads to the formation of amorphous and/or polycrystalline oxide on GaN [11, 12]. Therefore, in order to form " $\mathrm{N}-\mathrm{Ga}-\mathrm{O}-\mathrm{Zn}$ " bonding at the interface and to grow Zn-polar $\mathrm{ZnO}$ films on Ga-polar GaN, oxidation problems should be solved. On the other hand, in order to grow O-polar ZnO films on Ga-polar GaN templates, bonding sequences and hence polarity should be inverted.

We have examined two types of pre-growth treatments in order to control the interface bonding between the $\mathrm{ZnO}$ epilayer and $\mathrm{GaN}$ template: (1) $\mathrm{Zn}$ pre-exposure for to achieve Zn-polar growth, (2) O-plasma pre-exposure to form a monoclinic $\mathrm{Ga}_{2} \mathrm{O}_{3}$ interface layer, which should lead to the O-polar ZnO growth on the Ga-polar GaN template because it has a center of symmetry.

Figure 6 shows RHEED evolutions for the two types of pretreatments. Figures $6 \mathrm{a}$ and b show RHEED patterns before and after $\mathrm{Zn}$ pre-exposure on the GaN surface. As expected, RHEED did not show any appreciable change in terms of brightness and sharpness of the specular spot and the streaky rods, which indicates small adsorption of $\mathrm{Zn}$ as is the case for $\mathrm{Zn}$ adsorption onto a GaAs surface [13]. Figures $6 \mathrm{c}$ and d show RHEED patterns before and after O-plasma pre-exposure on the GaN surface. On exposing oxygen plasma onto a cleaned GaN surface without Zn pre-exposure, however, the RHEED pattern showed an appreciable change. A decrease in brightness and size of the specular spot, and a change in intensity of streaky rod were observed by O-plasma pre-exposure, which indicate a modification of the GaN surface by O-plasma pre-exposure.

The interface structure of the $\mathrm{ZnO} / \mathrm{GaN}$ heteroepitaxial layers has been investigated by HRTEM. The interface structure showed a drastic difference between the $\mathrm{ZnO}$ films with $\mathrm{Zn}$ and O-plasma pre-exposures. No interface layer was formed in between the GaN template and $\mathrm{ZnO}$ overlayer in the case of $\mathrm{Zn}$ pre-exposure (Fig. 7a), while an interface layer was observed for the O-plasma pre-exposure case (Fig. 7b). As shown in the inset of Fig. 7b, a diffraction pat- 

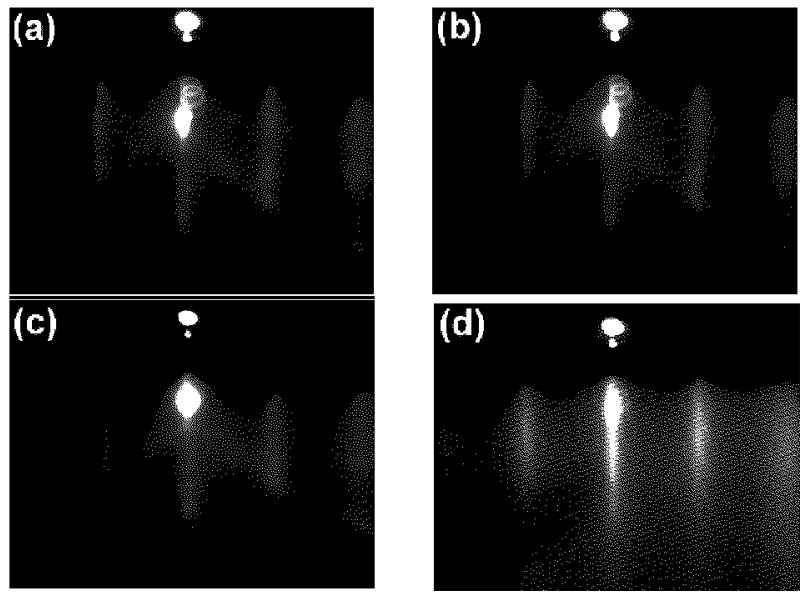

Fig. 6. RHEED patterns in $\langle 11 \overline{2} 0\rangle$ azimuth for two types of pretreatments on the GaN surfaces; (a) before and (b) after Zn pre-exposure; (c) before and (d) after O-plasma pre-exposure.
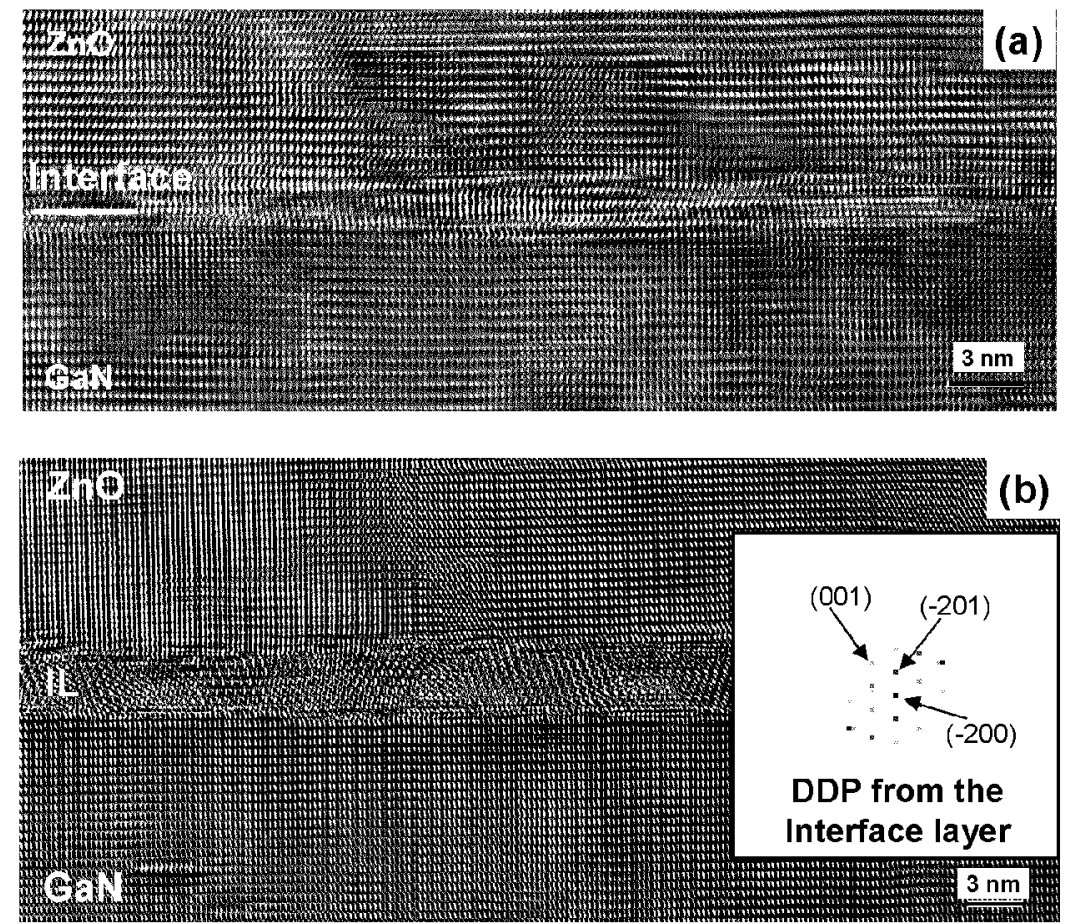

Fig. 7. HRTEM micrographs with the zone axis of [2 $\overline{110}]$ direction for $\mathrm{ZnO}$ films on Ga-polar GaN templates with (a) Zn pre-exposure and (b) O-plasma pre-exposure. The inset in (b) shows a diffraction pattern obtained from the interface layer. 
tern obtained by the Fourier transform of the interface layer image showed a single-crystalline pattern. The distances between the diffraction planes marked by the arrows are $0.52 \pm 0.01,0.53 \pm 0.01$ and $0.47 \pm 0.01 \mathrm{~nm}$. These interplane spacings can be assigned to the $(001),(\overline{2} 00)$, and (201) diffraction planes of monoclinic $\mathrm{Ga}_{2} \mathrm{O}_{3}$ and the zone axis can be assigned to [010]. A detailed analysis of the interface layer suggested that the interface layer was indeed of $\mathrm{Ga}_{2} \mathrm{O}_{3}$ [14]. The orientation relationship between $\mathrm{ZnO}, \mathrm{Ga}_{2} \mathrm{O}_{3}$, and $\mathrm{GaN}$ would be $\mathrm{ZnO}[2 \overline{11} 0] / \mathrm{Ga}_{2} \mathrm{O}_{3}[010] / \mathrm{GaN}[2 \overline{110}]$ and $\mathrm{ZnO}(0001) / \mathrm{Ga}_{2} \mathrm{O}_{3}(001) / \mathrm{GaN}(0001)$. It is noted that both interfaces at $\mathrm{ZnO} / \mathrm{Ga}_{2} \mathrm{O}_{3}$ and $\mathrm{Ga}_{2} \mathrm{O}_{3} / \mathrm{GaN}$ are sharp with small interface fluctuation.

We have further investigated possible in-plane atomic arrangements at the interface based on the observed orientation relationship using the unit cells of bulk $\mathrm{Ga}_{2} \mathrm{O}_{3}, \mathrm{GaN}$, and $\mathrm{ZnO}$. Figure $8 \mathrm{a}$ shows a $\mathrm{Ga}_{2} \mathrm{O}_{3}$ unit cell viewed along the [010] direction. A unit cell of $\mathrm{Ga}_{2} \mathrm{O}_{3}$ contains $8 \mathrm{Ga}$ and $12 \mathrm{O}$ atoms, and 10 fractional planes along the c-direction. Figure $8 \mathrm{~b}$ shows a projection view of two $\mathrm{Ga}_{2} \mathrm{O}_{3}$ unit cells along the direction normal to the (001) plane, in which one hexagonal unit cell of $\mathrm{GaN}$ (or $\mathrm{ZnO}$ ) is overlapped onto a $\mathrm{Ga}_{2} \mathrm{O}_{3}$ unit cell in accordance with the observed orientation relationship. In this schematic diagram, we have not considered in-plane or out-of-plane rearrangement of atoms at the interface. Although the lattice constant of $\mathrm{Ga}_{2} \mathrm{O}_{3}$ along the [010] direction $(0.304 \mathrm{~nm})$ is

(a)

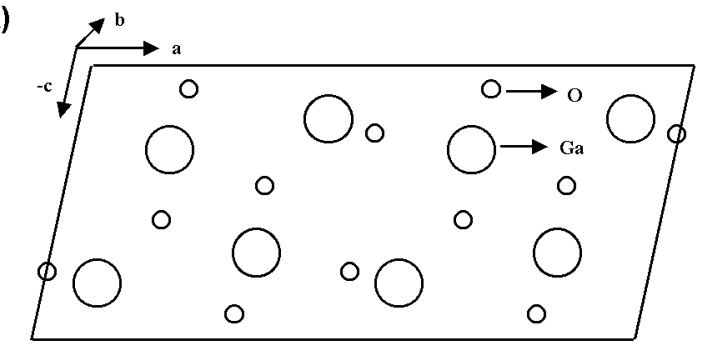

(b)

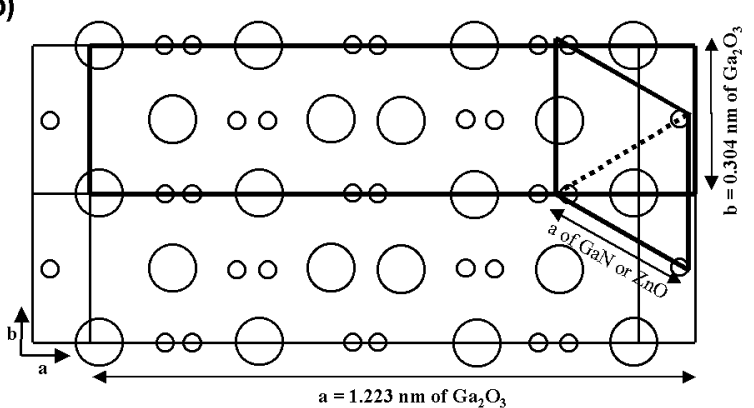

Fig. 8. (a) $\mathrm{Ga}_{2} \mathrm{O}_{3}$ unit cell viewed along the [010] direction. (b) Schematic diagram showing presumable in-plane lattice matching where $\mathrm{Ga}_{2} \mathrm{O}_{3}$ unit cell and hexagonal unit of $\mathrm{GaN}$ (or $\mathrm{ZnO}$ ) are overlapped. 
slightly smaller than the in-plane lattice constants of $\mathrm{GaN}(0.319 \mathrm{~nm})$ and $\mathrm{ZnO}$ $(0.325 \mathrm{~nm})$, the lattice constant of $\mathrm{Ga}_{2} \mathrm{O}_{3}$ along the [100] direction $(1.223 \mathrm{~nm})$ is about four times larger than the corresponding lattice parameters of $\mathrm{GaN}$ and $\mathrm{ZnO}$. Because of such large difference in the lattice constant between $\mathrm{Ga}_{2} \mathrm{O}_{3}$ and $\mathrm{ZnO}$ (or $\mathrm{GaN}$ ), one-to-one direct matching of the three unit cells is impossible. Instead, lattice matching of one unit of $\mathrm{Ga}_{2} \mathrm{O}_{3}$ along the [100] direction and four unit cells of $\mathrm{GaN}$ or $\mathrm{ZnO}$ along the [01 10 ] direction is possible considering domain matching epitaxy [15]. This situation may facilitate the epitaxial growth of single crystalline $\mathrm{ZnO}$ in spite of the large lattice misfit with the $\mathrm{Ga}_{2} \mathrm{O}_{3}$ interface layer. Thus the lattice misfits along the $[010](b=0.304 \mathrm{~nm})$ and $[100](a=1.223 \mathrm{~nm})$ axes of $\mathrm{Ga}_{2} \mathrm{O}_{3}$ with the $[2 \overline{110}](a=0.319 \mathrm{~nm})$ and $[01 \overline{1} 0](4 \sqrt{3} a / 2=1.105 \mathrm{~nm})$ directions of $\mathrm{GaN}$ are reduced to $-4.7 \%$ and $10.7 \%$, respectively. The corresponding lattice misfits between $\mathrm{Ga}_{2} \mathrm{O}_{3}$ and $\mathrm{ZnO}$ are $-6.5 \%$ and $8.6 \%$, respectively.

In definition, the polarity is caused by the lack of inversion center. If a crystalline layer whose crystal structure has an inversion symmetry is deposited on a surface of a Ga-polar GaN for example, it would be possible to interrupt the propagation of lattice polarity to the overlayer on the interface layer to invert the polarity. Here, it should be strongly noted that the monoclinic $\mathrm{Ga}_{2} \mathrm{O}_{3}$ interface layer has a center of symmetry, since the space group of $\mathrm{Ga}_{2} \mathrm{O}_{3}$ is $C 2 / m$. Therefore, the polarity of the $\mathrm{ZnO}$ film without any interface layer grown by $\mathrm{Zn}$ pre-exposure shall be Zn-polar. On the other hand, the polarity of the ZnO film with the monoclinic $\mathrm{Ga}_{2} \mathrm{O}_{3}$ interface layer shall be $\mathrm{O}$-polar, which implies polarity inversion in a wurtzite crystal from cation polar (Ga-polar GaN) to anion polar (O-polar).

The polarities of films and inversion of crystal polarities in the wurtzite structure using an interface layer with a center of symmetry are confirmed by direct determination of the polarities by CBED patterns. Figure 9a shows experimental CBED patterns of an upper $\mathrm{ZnO}$ film and a lower GaN one from a $\mathrm{Zn}$ pre-exposed sample. The arrow indicates the growth direction. The experimental CBED patterns from the upper $\mathrm{ZnO}$ and the lower $\mathrm{GaN}$ films are nearly the same. These indicate that the polarity of the upper $\mathrm{ZnO}$ film is of $\mathrm{Zn}$-polarity because we have used MOCVD grown Ga-polar GaN templates as substrates and the CBED patterns from $\mathrm{GaN}$ and $\mathrm{ZnO}$ are most likely very similar considering the structural similarity between $\mathrm{ZnO}$ and GaN. Figure $9 \mathrm{~b}$ shows experimental CBED patterns of an upper $\mathrm{ZnO}$ film and a lower GaN one from an O-plasma pre-exposed sample. Note that the experimental CBED patterns from the upper $\mathrm{ZnO}$ and the lower $\mathrm{GaN}$ films appear opposite. This implies the polarity of the $\mathrm{ZnO}$ film is opposite to that of the underlying $\mathrm{GaN}$, and hence the polarity of $\mathrm{ZnO}$ film is of O-polar. In order to determine the polarity without ambiguity, comparisons with simulated CBED patterns from $\mathrm{ZnO}$ and $\mathrm{GaN}$ are performed. Figure $9 \mathrm{c}$ shows simulated CBED patterns from wurtzite $\mathrm{ZnO}$ and GaN. The simulated patterns correspond to $28 \mathrm{~nm}$ and $45 \mathrm{~nm}$ thick $\mathrm{ZnO}$ and $\mathrm{GaN}$, in which the upward directions are aligned to $\mathrm{Zn}$ and Ga-polar, respectively. 

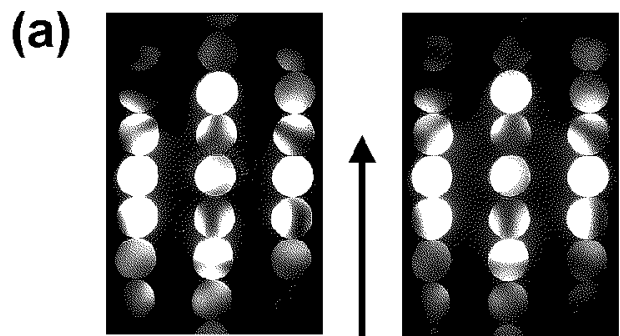

(b)
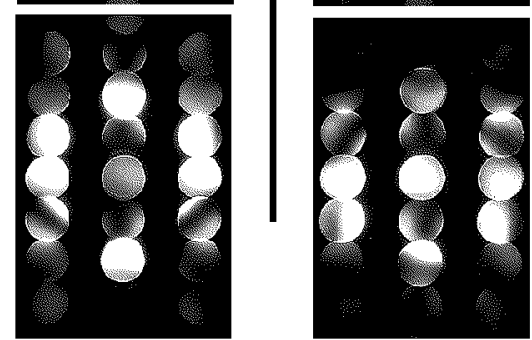

(c)

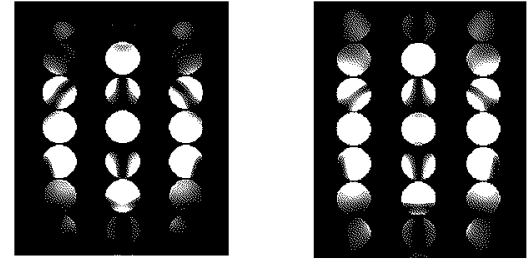

Fig. 9. Experimental CBED patterns of the upper $\mathrm{ZnO}$ and lower GaN films from (a) Zn pre-exposed sample and (b) O-plasma pre-exposed sample, and (c) simulated CBED patterns of wurtzite $\mathrm{ZnO}$ and $\mathrm{GaN}$ with the zone axis of [2 $\overline{110}]$ direction. The arrow indicates the growth direction. The simulated patterns correspond to $28 \mathrm{~nm}$ and $45 \mathrm{~nm}$ thicknesses of $\mathrm{ZnO}$ and $\mathrm{GaN}$, respectively, in which the upward directions are aligned to $\mathrm{Zn}$ and $\mathrm{Ga}$ polarities.

A comparison of the experimental and simulated CBED patterns directly indicates that the polarity of $\mathrm{ZnO}$ film with O-plasma pre-exposure is of O-polar and that of $\mathrm{ZnO}$ film with $\mathrm{Zn}$ pre-exposure is of $\mathrm{Zn}$-polar, which indicates that we have controlled polarities of wurtzite crystal by engineering the interfaces. We would like to stress again that the present results suggest a generalized method to control the lattice polarity of films by inserting an interface layer with a center of symmetry, thereby the conversion of lattice polarity becomes possible. It should be noted that the proposed method for controlling lattice polarity is more general than any of the previous methods using inverted domains $[16,17]$.

\section{Conclusions}

The importance of interface engineering in heteroepitaxy is demonstrated with examples of P-MBE $\mathrm{ZnO}$ epitaxy. The heterointerfaces in P-MBE grown 
$\mathrm{ZnO}$ films on sapphire and Ga-polar GaN template substrates are engineered to improve and to control the properties. The growth of rocksalt structure $\mathrm{MgO}$ buffer on $\mathrm{Al}_{2} \mathrm{O}_{3}(0001)$ is developed for $\mathrm{ZnO}$ epitaxy. Lattice misfit for $\mathrm{MgO}(111)$ / $\mathrm{Al}_{2} \mathrm{O}_{3}(0001)$ is $8.3 \%$, which implies accommodation of the lattice mismatch of $18.6 \%$ for $\mathrm{ZnO}(0001) / \mathrm{Al}_{2} \mathrm{O}_{3}(0001)$ by about half and half at $\mathrm{ZnO}(0001) / \mathrm{MgO}(111) /$ $\mathrm{Al}_{2} \mathrm{O}_{3}(0001)$ interfaces. By using the $\mathrm{MgO}$ buffer, the formation of $30^{\circ}$ rotated mixed domains is prohibited and two-dimensional layer-by-layer growth of $\mathrm{ZnO}$ on sapphire substrate is achieved. High-resolution X-ray diffraction (HRXRD) reveals the superior crystal quality of $\mathrm{ZnO}$ films with a $\mathrm{MgO}$ buffer.

$\mathrm{ZnO} / \mathrm{GaN}$ heterointerface without any interface layer is formed by the $\mathrm{Zn}$ pre-exposure on the GaN surface, which results in the growth of Zn-polar ZnO films on Ga-polar GaN templates. By the O-plasma pre-exposure on the Ga-polar GaN surface, single crystalline, monoclinic $\mathrm{Ga}_{2} \mathrm{O}_{3}$ interface layer is formed in between $\mathrm{ZnO}$ and $\mathrm{GaN}$. The polarity of $\mathrm{ZnO}$ films with the $\mathrm{Ga}_{2} \mathrm{O}_{3}$ interface layer is O-polar. The CBED result directly shows that the polarity of wurtzite structure is inverted from the cation polar (Ga-polar GaN) to the anion polar (Zn-polar $\mathrm{ZnO}$ ) by the formation of monoclinic $\mathrm{Ga}_{2} \mathrm{O}_{3}$, which has a center of symmetry.

\section{References}

[1] D.M. Bagnall, Y.F. Chen, Z. Zhu, T. Yao, S. Koyama, M.Y. Shen, T. Goto, Appl. Phys. Lett. 70, 2230 (1997)

[2] Z.K. Tang, G.K.L. Wong, P. Yu, M. Kawasaki, A. Ohotomo, H. Koinuma, Y. Segawa, Appl. Phys. Lett. 72, 3270 (1998).

[3] D.M. Bagnall, Y.F. Chen, Z. Zhu, T. Yao, M.Y. Shen, T. Goto, Appl. Phys. Lett. 73, 1038 (1998).

[4] H. Wenisch, V. Kirchner, S.K. Hong, Y.F. Chen, H.J. Ko, T. Yao, J. Crystal Growth 227-228, 944 (2001).

[5] Y. Chen, D.M. Bagnall, H.J. Ko, K.T. Park, K. Hiraga, Z. Zhu, T. Yao, J. Appl. Phys. 84, 3912 (1998).

[6] S.C. Jain, M. Willander, J. Narayan, R.V. Overstraeten, J. Appl. Phys. 87, 965 (2000).

[7] Y. Chen, H.J. Ko, S.K. Hong, T. Yao, Appl. Phys. Lett. 76, 559 (2000).

[8] Y. Chen, S.K. Hong, H.J. Ko, V. Kirshner, H. Wenisch, T. Yao, K. Inaba, Y. Segawa, Appl. Phys. Lett. 78, 3352 (2000).

[9] S.K. Hong, T. Hanada, H.J. Ko, Y. Chen, T. Yao, D. Imai, K. Araki, M. Shinohara, Appl. Phys. Lett. 77, 3571 (2000).

[10] S.K. Hong, T. Hanada, H.J. Ko, Y. Chen, T. Yao, D. Imai, K. Araki, M. Shinohara, K. Saitoh, M. Terauchi, Phys. Rev. B 65, 115331 (2002).

[11] S.D. Wolter, B.P. Luther, D.L. Waltemyer, C. Onneby, S.E. Mohney, R.J. Molnar, Appl. Phys. Lett. 70, 2156 (1997).

[12] S.D. Wolter, S.E. Mohney, H. Venugopalan, A.E. Wickenden, D.D. Koleske, J. Electrochem. Soc. 145, 629 (1998). 
[13] A. Ohtake, S. Miwa, L.H. Kuo, T. Yasuda, K. Kimura, C. Jin, T. Yao, J. Crystal Growth 164/185, 163 (1998).

[14] S.K. Hong, H.J. Ko, Y. Chen, T. Hanada, T. Yao, J. Vac. Sci. Technol. B 18, $2313(2000)$.

[15] T. Zheleva, K. Jagannadham, J. Narayan, J. Appl. Phys. 75, 860 (1994).

[16] V. Ramachandran, R.M. Feenstra, W.L. Samey, L. Salamanca-Riba, J.E. Northrup, L.T. Romano, D.W. Greve, Appl. Phys. Lett. 75, 808 (1999).

[17] L.T. Romano, J.E. Northrup, A.J. Ptak, T.H. Myers, Appl. Phys. Lett. 77, 2479 $(2000)$. 